\title{
Plasma membrane calcium pump: structure, function and relationships
}

\section{Journal Article}

\section{Author(s):}

Carafoli, E.

Publication date:

1997

Permanent link:

https://doi.org/10.3929/ethz-b-000422525

Rights / license:

In Copyright - Non-Commercial Use Permitted

\section{Originally published in:}

Basic Research in Cardiology 92, https://doi.org/10.1007/BF00794069 
Abstract The plasma membrane Ca-pump (134 kDa) is stimulated by calmodulin and by other treatments (exposure to acidic phospholipids, treatments with proteases, phosphorylation by protein kinases A or $C$, self-association to form oligomers). It is the product of four genes (in humans), but additional isoforms originate through alternative mRNA

Ernesto Carafoli (区)

Laboratory of Biochemistry III

Swiss Federal Institute of

Technology (ETH),

Universitätsstr. 16

8092 Zürich, Switzerland spicing. Most of the pump mass protrudes into the cytoplasm with three main units. The calmodulin binding domain is located in the C-terminal protruding unit. The domain is a positively charged segment of about 25 residues. The calcium-activated protease calpain activates the pump by removing its calmodulin binding domain and the portion C-terminal to it. The-resulting $124 \mathrm{KD}$ a fragment has been used to test the suggestion of an autoinhibitory function of the calmodulin binding domain. The latter interacts with two domains of the pump, one located close to the active site in the mid-cytoplasmic protruding unit, the other in the first
(N-terminal) protruding unit. The isoforms of the pump show variations in the regulatory domains, e.g., alternative mRNA splicing can eliminate the domain phosphorylated by protein kinase $\mathrm{A}$, or alter the sensitivity of the pump to calmodulin. This occurs by inserting sequences rich in His between calmodulin binding subdomains $\mathrm{A}$ and $\mathrm{B}$. The inserted domain(s) confer $\mathrm{pH}$ sensitivity to the binding of calmodulin. Calcium binding sites have been found in acidic regions preceding and following the calmodulin binding domain.

Key words Calcium pumpcalmodulin - calpain
The plasma membrane Ca-pump (PMCA) is the largest of all P-type ion pumps, i.e., pumps which form a phosphorylated intermediate in the catalytic cycle (an aspartyl-phosphate) and are inhibited by vanadate. The PMCA pump interacts with $\mathrm{Ca}$ with high affinity, but has a low total Ca transporting capacity. This property makes it inadequate to rapidly eject large amounts of $\mathrm{Ca}$, as required, for exampie, by the functional cycle of heart cells. Thus, in these cells, the pump is quantitatively overshadowed by a larger system, the $\mathrm{NaCa}$ exchanger. First discovered in erythrocytes, the PMCA pump has been subsequently found in many other cells, and is now assumed to be an obligatory component of eucaryotic plasma membranes. It is stimulated by calmodulin and, in its absence, by a number of alternative treatments like the exposure to acidic phospholipids (including the phosphorylated derivatives of phosphatidyl-inositol), a controlled treatment with proteases, e.g., trypsin or calpain, phosphorylation reactions by protein kinases $\mathrm{A}$ or $\mathrm{C}$ and self-association to form dimers or, more likely, oligomers.

Cloning work $(4,8)$ in the late 1980 s has shown that the pump is a single polypeptide chain of molecular mass about $134 \mathrm{KDa}$. It is the product of a multigene family: four genes have been recognized in humans, and they have been assigned to chromosomes $1,3,12$ and $x$. Numerous additional isoforms then originate through alternative mRNA spicing. The general architecture of the pump (Fig. 1) follows the general pattern of the P-type pump family, i.e., most of the pump mass protrudes into the cytoplasmic medium, very short loops connecting the 
putative transmembrane domain on the external side. Ten putative transmembrane domains have been identified, four concentrated in the $\mathrm{N}$-terminal portion of the pump and six in the C-terminal portion. The transmembrane organization of the pump has been, to a large part, directly validated by work with monoclonal antibodies and with other chemical labelling techniques. Three main units protrude into the cytosol, the first between transmembrane domains 2 and 3 , the second between transmembrane domains 4 and 5 , and the third from transmembrane domain 10 . The first protruding unit has been proposed, based on the analogy with other P-type pumps, to permit the coupling of ATP hydrolysis to the transport of $\mathrm{Ca}$ and is thus sometimes called the transducing unit. The protruding portions of the pump (Fig. 1) consist of antiparallel $\beta$-sheets, a-helices and parallel $\beta$-sheets. The domain protruding between transmembrane segments 4 and 5 contains the active site(s) of the pump (i.e., the site of aspartyl-phosphate formation and the binding site for ATP), and is assumed to be connected to the membrane by a flexible "hinge" in its C-terminal portion which permits the catalytic aspartic acid to approach the bound ATP during the reaction cycle.

The calmodulin binding domain of the pump has been identified with the help of bifunctional crosslinkers coupled to calmodulin (James et al., 1988), and located in

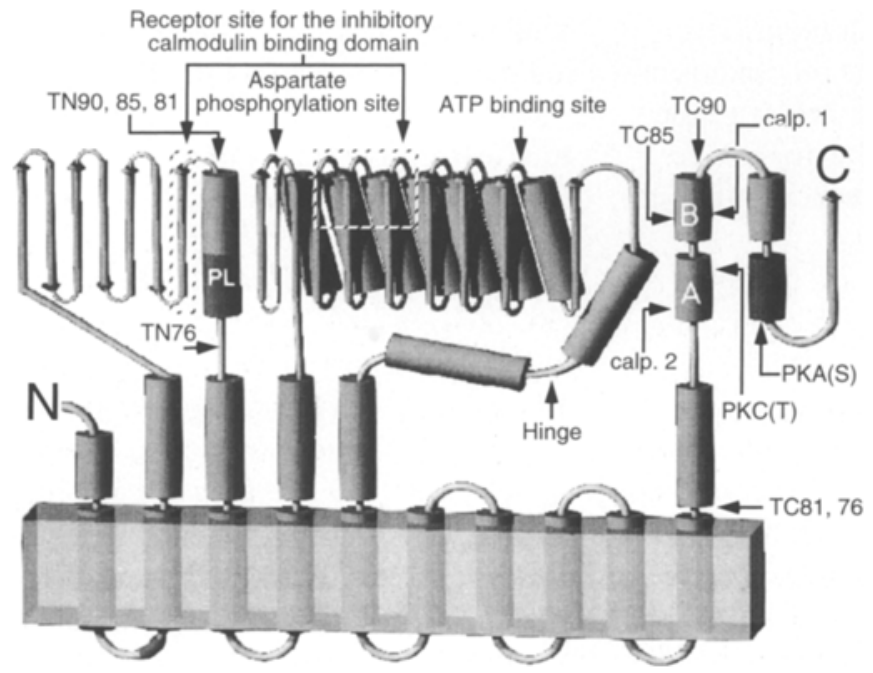

Fig. 1 Membrane architecture and domain definition of the PMCA pump. Cylinders indicate predicted $\alpha$-helices, arrows $\beta$-sheets. The two calmodulin binding sub-domains are indicated by A and B. Calp 1 and calp 2 are the two sequential sites of calpain cleavage. PKA (S) and PKC (T) are sites of serine and threonine kinase phosphorylation: PKA only phosphorylates one of the isoforms of the pump. TC is the notation for the $\mathrm{C}$-terminal cleavage by trypsin, TN that of the $\mathrm{N}$-terminal cleavage. $\mathrm{PL}$ is the main domain responsive to acidic phospholipids. the last protruding unit of the pump. The domain is a positively charged segment of about 25 residues, which has a strong $\alpha$-helix propensity in its $\mathrm{N}$ - and $\mathrm{C}$-terminal portions (sub-domains A and B). Work with trypsin has shown that the pump is sequentially degraded to fragments of electrophoretic molecular masses $90,85,81,76 \mathrm{KDa}$. The 90 $\mathrm{KDa}$ fragment has low basal activity and is stimulated by both calmodulin and acidic phospholipids. During the formation of the 85 and $81 \mathrm{KDa}$ fragments, the pump gradually loses its sensitivity to calmodulin, but retains that to acidic phospholipids, whereas the formation of the 76 KDa fragment is accompanied by the loss of phospholipid sensitivity as well. The removal of the calmodulin binding domain and of the pump portion C-terminal to it during the formation of these fragments leads to the stimulation of the basal activity of the pump, suggesting that the domain acts as an autoinhibitor of the pump. In the formation of the 90 to $76 \mathrm{KDa}$ fragments, trypsin also cuts the pump between putative transmembrane domains 2 and 3 , but it is doubtful whether the portion of the pump N-terminal to the cut is actually removed. The Ca-dependent protease calpain also activates the pump, and does so by removing in two steps its calmodulin binding domain and the portion C-terminal to it. The resulting $124 \mathrm{KDa}$ fragment has been used to test the suggestion of the autoinhibitory function of the calmodulin binding domain. The latter, suitably derivatized with a photoactivatable crosslinker (2) has been found to label two domains of the pump, one located between the sites of aspartyl-phosphate formation and of ATP-binding, the other in the first protruding unit of the pump. The finding that the "receptor" sites for the calmodulin binding domain are located in close proximity to the active site(s) of the pump conveniently rationalizes the "inhibitory" action of the domain. The calmodulin binding domain can be phosphorylated by protein kinase $\mathrm{C}$, leading to some activation of the basal activity of the pump (9). The calmodulin-binding domain has been prepared synthetically with a phosphate on the threonine that is the substrate for protein kinase $\mathrm{C}$ (6): the phosphorylated domain is unable to inhibit the fully active $124 \mathrm{kDa}$ calpain product. This establishes a striking parallel with the Ca-pump of sarcoplasmic reticulum which is kept inhibited by the association of the accessory protein, phospholamban, and which is reactivated by the removal of phospholamban from its binding site by kinase-directed phosphorylations.

On the $\mathrm{N}$-terminal side, trypsin cleaves the pump between transmembrane domains two and three. The $\mathrm{N}$ termini of the fragments of $90,85,81 \mathrm{KDa}$ differ from that of the $76 \mathrm{KDa}$ fragment by a heavily charged stretch of about 40 residues. Work with the synthetic $\mathrm{C}$-terminal portion of the stretch, which is very rich in basic amino acids (labelled PL in Fig. 1), has shown (1) it to be one of the 
sites of the pump responsible for the response to acidic phospholipids (the other site appears to be the calmodulin binding domain itself).

None of the isoforms of the pump show variations in the domains which are preserved throughout the family of P-type ion motive ATPases, e.g., the domains surrounding the active site(s). Most of the diversity concerns the regulatory domains and leads to differences in regulatory properties, e.g., alternative mRNA splicing can eliminate the domain phosphorylated by protein kinase A $(\mathrm{PKA}(\mathrm{S})$ in Fig. 1). A particularly interesting mRNA splicing process leads to inserts of increasing length between calmodulin binding subdomains $\mathrm{A}$ and $\mathrm{B}$. The newly inserted domain(s) duplicate somewhat the original calmodulin binding domain, except that the positively charged amino acids are histidines instead of arginines and lysines. This confers to the newly inserted domain(s) $\mathrm{pH}$ sensitivity in the binding of calmodulin, and may thus endow the pump with additional modulation possibilities.

One domain of the pump which is still unknown is that containing the catalytic $\mathrm{Ca}$ binding site. By analogy with suggestions on the Ca pump of sarcoplasmic reticulum, this Ca binding site could be located within some of the transmembrane domains. Mutagenesis work on selected residues in some of the transmembrane domains are in line with the prediction. Other $\mathrm{Ca}$ binding sites, possibly regulatory, have recently been found immediately $\mathrm{C}$ - and $\mathrm{N}$-terminal to the calmodulin binding domain (6).

Despite the similarity of the plasma membrane and the endoplasmic reticulum Ca pumps, the two enzymes are targeted to two different membranes. Chimeric constructs of the two proteins have shown that the region encompassing the first two transmembrane domains of the SERCA pump contains a strong endoplasmic reticulum retention signal. Chimeric constructs in which the first two transmembrane domains of the PMCA pump are followed by the remainder of the SERCA pump are still largely retained in the reticulum: i.e., the SERCA pump contains additional endoplasmic reticulum retention signals elsewhere in the molecule that outweigh those present in the first two PMCA transmembrane domains (3).

\section{References}

1. Brodin $P$, Falchetto $R$, Vorherr $T$, Carafoli E (1992) Identification of two domains which mediate the binding of activating phospholipids to the plasma membrane $\mathrm{Ca}^{2+}$ pump. Eur J Biochem 204: 939-946

2. Falchetto $\mathrm{R}$, Vorherr $\mathrm{T}$, Brunner $\mathrm{J}$, Carafoli $\mathrm{E}$ (1991) The plasma membrane $\mathrm{Ca}^{2+}$ pump contains a site that interacts with its calmodulin binding domain. I Biol Chem 266: 2930-2936

3. Foletti D, Guerini D, Carafoli E (1995) Subcellular targeting of the endoplasmic reticulum and plasma membrane $\mathrm{Ca}^{2+}$ pumps: a study using recombinant chimeras. FASEB J 9: 670 -680
4. Greeb J, Shull GE (1989) Molecular cloning of a third isoform of the calmodulinsensitive plasma membrane $\mathrm{Ca}^{2+}$-transporting ATPase that is expressed predominantly in brain and skeletal muscle. $J$ Biol Chem 264: 18569-18576

5. Hofmann F, Anagli I, Carafoli E, Vorherr T (1994) Phosphorylation of the calmodulin binding domain of the plasma membrane $\mathrm{Ca}^{2+}$ pump by protein kinase $\mathrm{C}$ reduces its interaction with calmodulin and with its pump receptor site. J Biol Chem 269: 24298-24303

6. Hofmann F, James P, Vorherr T, Carafoli E (1993) The C-terminal domain of the plasma membrane $\mathrm{Ca}^{2+}$ pump contains three high affinity binding sites. J Biol Chem 268: 10252-10259

7. James P, Maeda M, Fischer R, Verma AK, Krebs J, Penniston JT, Carafoli E (1988) Identification and primary structure of a calmodulin-binding domain of the $\mathrm{Ca}^{2+}$ pump of human erythrocytes. $\mathrm{J}$ Biol Chem 263: 2905-2910
8. Verma AK, Filoteo AG, Stanford RD, Wieben ED, Penniston JT, Strehler EE, Fischer R, Heim R, Vogel G, Mathews S, Strehler-Page, M-A, James P, Vorherr T, Krebs J, Carafoli E (1988) Complete primary structure of a human plasma membrane $\mathrm{Ca}^{2+}$ pump. J Biol Chem 263: 14152-14159

9. Wang $\mathrm{KKW}$, Wright LC, Machan $\mathrm{CL}$, Allen $\mathrm{BC}$, Conigrave AD, Roufogalis BD (1991) Protein kinase C phosphorylates the carboxyl terminus of the plasma membrane $\mathrm{Ca}^{2+}$-ATPase from human erythrocytes. J Biol Chem 266: 90789085 